\title{
Towards a Metric Between Engineering to Order and Assemble/Make to Order Products in Configuration Situations
}

\author{
A. Sylla ${ }^{1,3}$, R. Ayachi ${ }^{2,3}$, M. Aldanondo ${ }^{2,4}$, E. Vareilles ${ }^{2,4}$, Y. Beauregard ${ }^{4}$, P. Pitiot ${ }^{2,5}$ \\ ${ }^{1}$ Université Grenoble Alpes - G-SCOP, Grenoble, France \\ ${ }^{2}$ Université de Toulouse - IMT Mines Albi-CGI, Albi, France \\ ${ }^{3}$ Université de Toulouse - INP ENI Tarbes-LGP, Tarbes, France \\ ${ }^{4}$ Mechanical Engineering, École de Technologie Supérieure, Montréal, Canada \\ ${ }^{5}$ Institut d'Ingénierie Informatique de Limoges, Rodez, France
}

\begin{abstract}
Most companies that supply customized or configured product use product configuration systems (PCS). Especially in B2B for technical systems, it happens frequently that the supplier and his PCS cannot find a solution that fulfills all customer requirements because some of them are out of the company standard. In that case, if the supplier wants to make an offer, it is necessary to perform some engineering activities in order to propose a solution which fulfills the out of standard requirements. The key questions that come to the supplier in these situations are: Do we accept this out of standard demand? How far is it from our standard? What are the risks? The goal of this paper is to propose new metrics that can help to answer these questions.
\end{abstract}

\section{Keywords - Configuration, ETO products, ETO-Level}

\section{INTRODUCTION}

This paper deals with product configuration or customization. Configuration is a kind of design activity where products are defined given predefined sets of components linked by compatibility constraints [1]. It is supported by software tools generally called Product Configuration Systems (PCS).

A PCS gathers two main parts: (i) a knowledge base which contains a generic model of the products and (ii) a processing unit that interacts with the user in order to assist her/him during the configuration activity. The generic model contains all component families and all compatibility constraints between components. One generic model represents a family of products with all its possible options and alternatives. The processing unit is basically responsible of constraint propagation. This means that for each requirement inputted by the user, the constraints are propagated by the processing unit and the set of possible solutions of each component family is reduced accordingly. For more details, consult [1].

In this article, we consider the configuration of mechatronics technical systems (e.g. machine tool, robots, cranes and buses) in a business to business (B2B) customer/supplier relationship. Moreover, we assume that the supplier has setup a PCS and is working mainly in Assemble/Make-To-Order (AMTO) or Configure-ToOrder (CTO) industrial situations. This means that the supplier has defined all the generic models of the configurable products she/he wants to sell on the market. This assumes that she/he has studied and decided about the allowed product diversity and/or product range which correspond to what we call the "standard offer" of the company. But very frequently, especially in B2B, customers require products that are a little bit "out of standard". In such a case, in order to study and fulfill the "out of standard" requirements, some engineering works are required. Therefore, we are not any more in CTO but in Engineering-To-Order (ETO) situations [2]. Three questions come to the supplier. The first one is: Do we accept this out of standard demand? The questions that follow are: How far are we from the standard? What are the risks?

Therefore, the goal of this article is to provide the first elements that allow suppliers to answer these questions. A new metric, named "ETO-Level" or "ETOL", is proposed. It allows to quantify a kind of distance between an out of range product (a non-standard/ETO product) and a standard/CTO one.

The remaining of the article is as follow. Section II discusses some background about configuration and ETO industrial situations. It also provides the generic model that will be used to illustrate our proposals. Then, section III presents our proposal in four main points. The last section presents conclusion and further research.

\section{CONFIGURATION, ETO, GENERIC MODEL}

\section{A. Configuration background}

Following previous works achieved by the main authors of the configuration domain as Mittal and Frayman [3], Soininen et al. [4], Vareilles et al. [5] or Yang and Dong [6], we consider the following formal definition of a product configuration.

Hypothesis: a product is defined as a set of components.

Given: (i) a generic architecture of the product that describes a family of products, (ii) a fixed set of component groups, (iii) a fixed set of properties or attributes that characterize either a component or a product, (iv) a set of constraints that restrict possible combinations of components and/or property values (v) a set of customer requirements, where a requirement corresponds to the selection of a component in a group or a value for a property.

The configuration of a product consists in finding at least one set of components that satisfies all the constraints and the customer requirements.

As noticed in [2],[7] neither non-standard components nor non-standard components integrations can be selected 
to configure a non-standard technical system. Configuration problem hypotheses require staying inside the set of the standard solutions. Therefore, ETO product in this case, this item has no decomposition. When they are not bought, the part item PA is manufactured and assembled while all other items (except CP and RM that

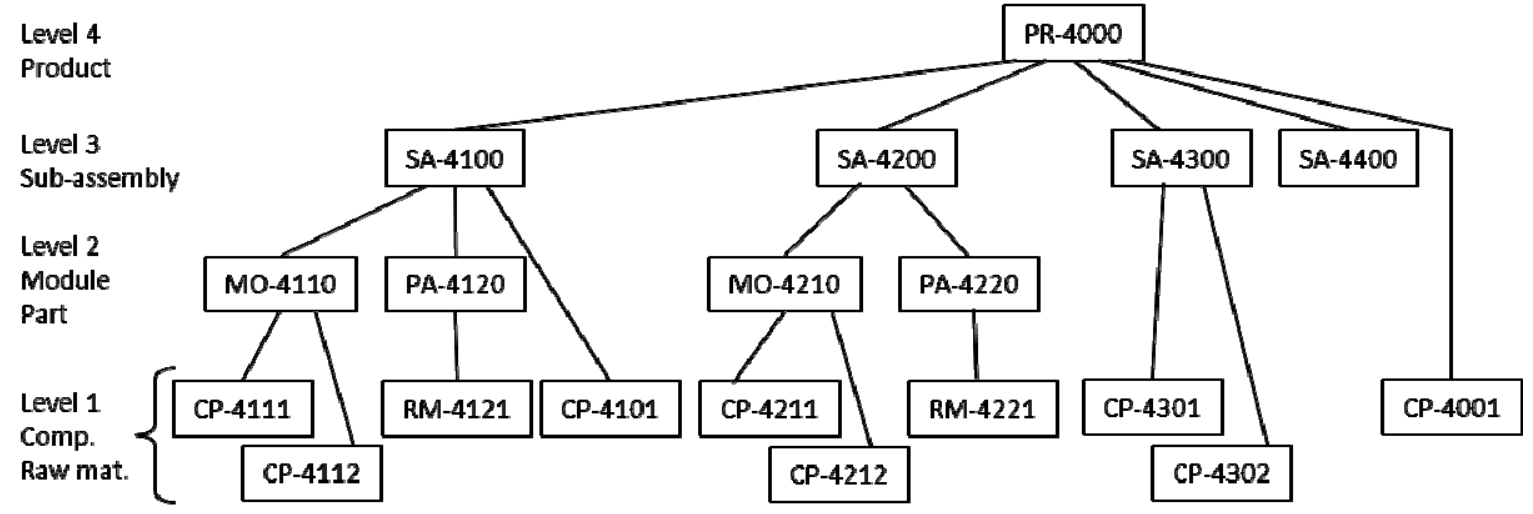

Figure 1 - Configuration Generic model as Generic bill-of-materials

configuration cannot be supported by a PCS in line with previous ATO/MTO hypotheses and definitions.

In the literature, many studies have been reported in the field of ATO/MTO product generic modeling, see [4],[8],[6] or [9]. However, much fewer concern ETO products, see [7],[10]. While most of these papers addresses knowledge modeling, they concern mainly either CTO or ETO products and do not attempt to bridge the gap between them. Moreover, many metrics have been proposed to characterize diversity and commonality, see the survey in [11]. However, only a recent paper [7] proposes first ideas that deal with the identification of various types of differences between ETO and CTO products. They concern the existence of (i) non-standard component in a group, (ii) non-standard group of components in a product, (iii) non-standard integration of components in a product. The authors also introduce a notion of heavy or light ETO but they did not provide any metrics to quantify the ETO level of a non-standard product comparatively to a standard one.

Therefore, the goal of this article is to provide the first ideas about metrics which allow to quantify the differences between an ETO and a CTO product.

\section{B. Product representation and generic model}

In order to clearly explain and illustrate our proposals, we need a configuration generic model. To be more realistic, we consider a model with intermediate decomposition levels. Consequently, we assume the following four-level product representation (see Fig. 1).

- Product $(\mathrm{PR})=$ set of Sub-assemblies $(\mathrm{SA})$ and components (CP),

- Sub-assembly (SA) = set of modules (MO), parts (PA) and components $(\mathrm{CP})$,

- Module $(\mathrm{MO})=$ set of components $(\mathrm{CP})$

- Part (PA) = set of raw materials (RM)

This representation is therefore based on six items: Products (PR), Sub-assemblies (SA), Modules (MO), Parts (PA), Components (CP) and Raw materials (RM). Any item, product excepted, can be bought on the market; are always bought) are only assembled.

Given previous product representation and as we assume a PCS running CTO products configuration, all the allowed items and their combinations exist and are defined in a configuration generic model of the product. This generic model corresponds to a generic hierarchical bill of materials (noted G-BOM) that is based on generic items (noted G-Item). This means that each item represents a family of items organized as follow:

- G-PR (Product) = set of: G-SA (Sub-assemblies), G-CP (components), G-PR-attributes

- G-SA (Sub-assemblies) = set of: G-MO (modules), GPA (parts), G-CP (components), G-SA-attributes

- G-MO (modules) = set of: G-CP (components), G-MOattributes

- G-PA (part) = set of: G-RM (raw materials and manufacturing process), G-PA-attributes

- G-CP (components) and G-PA (parts) = set of: G-CPattributes and G-RM-attributes.

Constraints limit the possible combinations of GItems and/or G-Item attributes. We consider as notation, that product level is 4 , sub-assembly level is 3 , module and part level is 2 while component and raw material level is 1 . Any generic item of level " $i$ " is characterized by a set of variables that gather the generic items names of the lower levels " $<\mathrm{i}$ " and attributes that describe the generic item of level "i".

Any generic item without decomposition which is bought has among its descriptive attributes, two specific attributes: its supplier and cost. An example of generic model is shown in Fig. 1. This example will be used with further metric computations.

\section{TOWARD ETO METRICS PROPOSITIONS}

Some metrics requirements have been identified. First the metric should be independent of the considered technical aspect of the product, by independent we mean that the metric depends only on previous G-BOM. Furthermore, ETO level is a quote between 0 to 1000 , where a 0 value means that the customer requirements 
respect the standard and no engineering is required, while 1000 means that all requirements are out of standard and that the product must be entirely re-engineered. It is clear that we are more interested in quantifying the situations rather close to the standard, because we assume an ATOMTO situation running a PCS.

\section{A. First raw physical item approach}

The key idea is based on the following. In the introduction, we said that when a customer requires an out of standard product, the questions that come to the supplier are: Do we accept? How far are we from the standard and what are the risks? We assume now that the engineering team of the supplier company is able to answer "We should re-design, modify and/or change this generic item, this generic item and this generic item...." Re-designing or changing a G-item means adding: (i) a new value in the definition domain of a G-item attribute, (ii) a new possible item in a lower level G-item or, (iii) a new allowed combination of items in a constraint.

Given our generic model, we have a four levels tree structure of items. It is therefore possible to map engineering demand or the generic items that need to be changed on the G-BOM. We assume in this first approach that there is a top-down change propagation, meaning that if a high-level item must be changed there must be at least a change in one of the existing lower level items. So, this distance cannot deal with changes that correspond to "pure" item integration without item modification. This will be discussed later in section $\mathrm{D}$.

ETO-level is computed as follow:

- 1 Map the engineering demand on the G-BOM,

- 2 For any G-item of level "i", calculate the change ratio of direct lower level G-items:

o Count the total number of direct lower level G-items " $<\mathrm{i}$ ", noted Nbit-Lv $<\mathrm{i}$

○ For each direct lower level G-item of level " $<$ i", the change ratio, noted $\mathrm{Chr}-\mathrm{Lv}<\mathrm{i}$, is equal to:

- 1 / Nbit-Lv<i, if the G-item must be changed

- 0 , if the G-item is unchanged

Direct lower level G-items " $<\mathrm{i}$ " can correspond with:

- for "i” = 4 (PR): SA (level 3), CP (level 1),

- for “i” = 3 (SA): MO or PA (level 2), CP (level 1),

- for "i" = 2 (MO): CP (level 1),

- for "i" = 2 (PA): RM (level 1),

- 3- Calculate the ETO level of the product as the sum on all the branches of the G-BOM of the product of all ratio of change starting at the lowest level. If a G-BOM link skip a level (bought item or direct link with high and low levels), count 1 for the missing change ratio.

$$
\text { ETO-L }=1000 * \Sigma\left(\mathrm{Chr}_{-\mathrm{Lv}_{1}} * \mathrm{Chr}-\mathrm{Lv}_{2} * \mathrm{Chr}_{\mathrm{L}} \mathrm{Lv}_{3}\right)
$$

For example, on the case of figure 1, let us assume that the generic items that should be changed are: SA-4100, MO-4110, CP-4111, SA-4400, CP-4001. This provides the following change ratio:

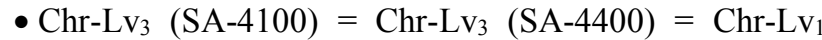
$(\mathrm{CP}-4001)=1 / 5$

- $\mathrm{Chr}-\mathrm{Lv}_{2}(\mathrm{MO}-4110)=1 / 3$

- $\mathrm{Chr}-\mathrm{Lv}_{1}(\mathrm{CP}-4111)=1 / 2$

And the following ETO-Level:

- ETO-L $=1000 *(1 / 2 * 1 / 3 * 1 / 5+1 * 1 * 1 / 5+1 / 5 * 1 * 1)$

- $\mathrm{ETO}-\mathrm{L}=33.3+200+200=433 \%$

This value can be roughly interpreted as almost half of the product must be re-engineered.

The immediate drawback of this approach is that the abstraction level of a bought item is not taken into account. In other words, in our example, SA-4400 which can be a main sub-assembly (as a big engine) has the same impact as CP-4001 which could be a simple assembling component (as a screw). This induces a second definition object of the next section.

\section{B. Second approach considering abstraction level}

In order to consider abstraction level this second metric is an update of the previous one as follows:

- Each change ratio is multiplied by 10 , which gives for the new value of $\mathrm{Chr}-\mathrm{Lv}<\mathrm{i}$

○ $10 / \mathrm{Nbit}-\mathrm{Lv}<\mathrm{i}$, if the G-item must be changed

$\circ 0$, if the G-item is unchanged

- ETO-Level calculation is similar to the previous approach except that:

o If a G-Item of level 2 or 3 is not decomposed (meaning it is bought), a fictive decomposition until level 1 is assumed with $\mathrm{Chr}-\mathrm{Lv}<\mathrm{i}=10$ for each level.

o If an intermediate level G-Item is skipped (direct link with high and low levels), a fictive item is assumed with $\mathrm{Chr}-\mathrm{Lv}<\mathrm{i}=1$ for each level.

Considering the example of section $A$, this second approach provides the following change ratio:

- $\mathrm{Chr}-\mathrm{Lv}_{3}(\mathrm{SA}-4100)=\mathrm{Chr}_{-} \mathrm{Lv}_{3}(\mathrm{SA}-4400)=\mathrm{Chr}_{-} \mathrm{Lv}_{1}(\mathrm{SA}-$ $4001)=10 / 5$

- $\mathrm{Chr}_{-} \mathrm{Lv}_{2}(\mathrm{MO}-4110)=10 / 3$

- $\mathrm{Chr}-\mathrm{Lv}_{1}(\mathrm{CP}-4111)=10 / 2$

And the relevant ETO-Level:

- $\mathrm{ETO}-\mathrm{L}=(10 / 2 * 10 / 3 * 10 / 5+10 * 10 * 10 / 5+10 / 5 * 1 * 1)$

- $\mathrm{ETO}-\mathrm{L}=33.3+200+2=235.3 \%$

This strong decrease of the ETO-L value (from 433 \%o for the first approach to $235.3 \%$ for the second one) results from the new branch product calculation that provides for last component CP-4111 a value of 10/5 compared with the value $1000 / 5$ of the sub-assembly SA4400. The abstraction levels of the bought items are now strongly considered.

The drawback of this second approach is that it considers that for any item at any level, their importance between two levels is uniformly distributed. For example, for PR-4000 the four sub-assemblies SA-4100, SA-4200, SA-4300 and SA-4400 have roughly the same importance or impact on PR-4000 characteristics (performance, 
cost...). Next section will propose something that can handle this problem.

\section{Third physical approach considering item importance}

The idea is to modify the change ratio calculation of lower level G-Items. Instead of simply counting the quantity of components, we propose to replace the $0 / 1$ value by a "relative importance" value for each G-item. As it is quite difficult to quantify the importance of a Gitem in an assembly, we propose to consider its cost as a representation of its importance. For any level, the cost of a G-item is the sum of the cost of lower level G-items plus the processing cost which is mainly assembling (only parts have some manufacturing). The delicate point of this idea is that the cost of a G-Item can vary a lot (think for example about different engines for large and small buses). For a first approach, we consider that the cost of a generic item (noted Cs-G-Item) equals the average of the cost of all configured items that belongs to this Generic item. As the expected importance is a relative notion, we consider that this assumption is not too strong. The ETOLevel calculation goes therefore as follow.

- 1 Map the engineering demand (as before)

- 2 For any G-item of level "i", calculate its change ratio: o Calculate the total cost of direct lower level G-items " $<\mathrm{i}$ ", noted $\mathrm{Cs}-\mathrm{Lv}<\mathrm{i}$, that equals $\Sigma$ Cs-G-Item

o For each lower level G-items of level " $<i$ ", the change ratio, noted $\mathrm{Chr}-\mathrm{LV}<\mathrm{I}$, is equal to:

- Cs-G-Item/ Cs- $\mathrm{Lv}_{<i}$, if the G-item must be changed

- 0 , if the G-item is unchanged

- 3 Calculate the ETO exactly as in section 3.1

ETO-L $=1000 * \Sigma\left(\mathrm{Chr}_{-} \mathrm{Lv}_{1} * \mathrm{Chr}-\mathrm{Lv}_{2} * \mathrm{Chr}-\mathrm{Lv}_{3}\right)$

The example of Fig. 1 is considered again with the previous costs on Fig. 2. Inside a G-item box is the Gitem average cost, under the item is the average processing cost. For any G-item, its cost equals the sum of direct lower level G-items costs plus its processing cost.

Assuming the same required changes: SA-4100, MO4110, CP-4111, SA-4400, CP-4001. This provides the following change ratio:

- $\mathrm{Chr}_{-L_{3}}(\mathrm{SA}-4100)=400 / 800, \mathrm{Chr}_{-L_{3}}(\mathrm{SA}-4400)=$ 200/800, Chr-Lvi $($ SA-4001) $=20 / 800$

- $\mathrm{Chr}_{-} \mathrm{Lv}_{2}(\mathrm{MO}-4110)=150 / 300$
- $\mathrm{Chr}_{-\mathrm{Lv}_{1}}(\mathrm{CP}-4111)=50 / 100$

And the following ETO-Level:

- $\mathrm{ETO}-\mathrm{L}=1000 *(50 / 100 * 150 / 300 * 400 / 800+$ $1 * 1 * 200 / 800+20 / 800 * 1 * 1)$

- $\mathrm{ETO}-\mathrm{L}=125+250+25=400 \%$

The importance of sub-assembly SA-4100 is clearly considered (125 compared to 33 with the previous ones). Another point is that it integrates the abstraction level of G-Item with their cost (a factual information) without any specific consideration in calculations.

\section{About integration and change types.}

This section discusses two important issues that have not been investigated in detail in the previous sections.

The first one is the engineering of new integrations between G-items. It occurs in situations where a company need to re-design a given G-Item without modifying its lower level G-Items. For example, let us consider the GItem MO-4110 with two lower levels G-items CP-4111 and CP-4112 which both gathers two existing physical items: CP-4111_1, CP-4111_2 and CP-4112_1, CP4112_2. Let us also consider that all combinations (with their integration), except (CP-4111_2, CP-4112_2), have been fully designed and used to assemble various physical items of $\mathrm{M} 0-4110$. If a specific customer requirement corresponds to this last combination (CP-4111_2, CP4112 2), as the integration of the two physical items has not been designed yet, it will be necessary to perform some engineering activities in order to achieve this assembly. There is no design or engineering activity on the lower level G-items but on their integration.

Two ideas may be considered to take into account the engineering of integration between lower level G-Items in the computation of the ETO-L of a G-Item. The first one follows the ideas of the computation of the technological maturity or readiness of a complex system as proposed in [12]. It consist of adding to the G-BOM generic integration components (with a cost). Then, with some aggregation method, to include the cost of the integrations in the computation of the change ratio of a G-Item. The second one is to consider that the engineering of integrations is part of the assembling process. Consequently, the processing cost introduced in section $C$

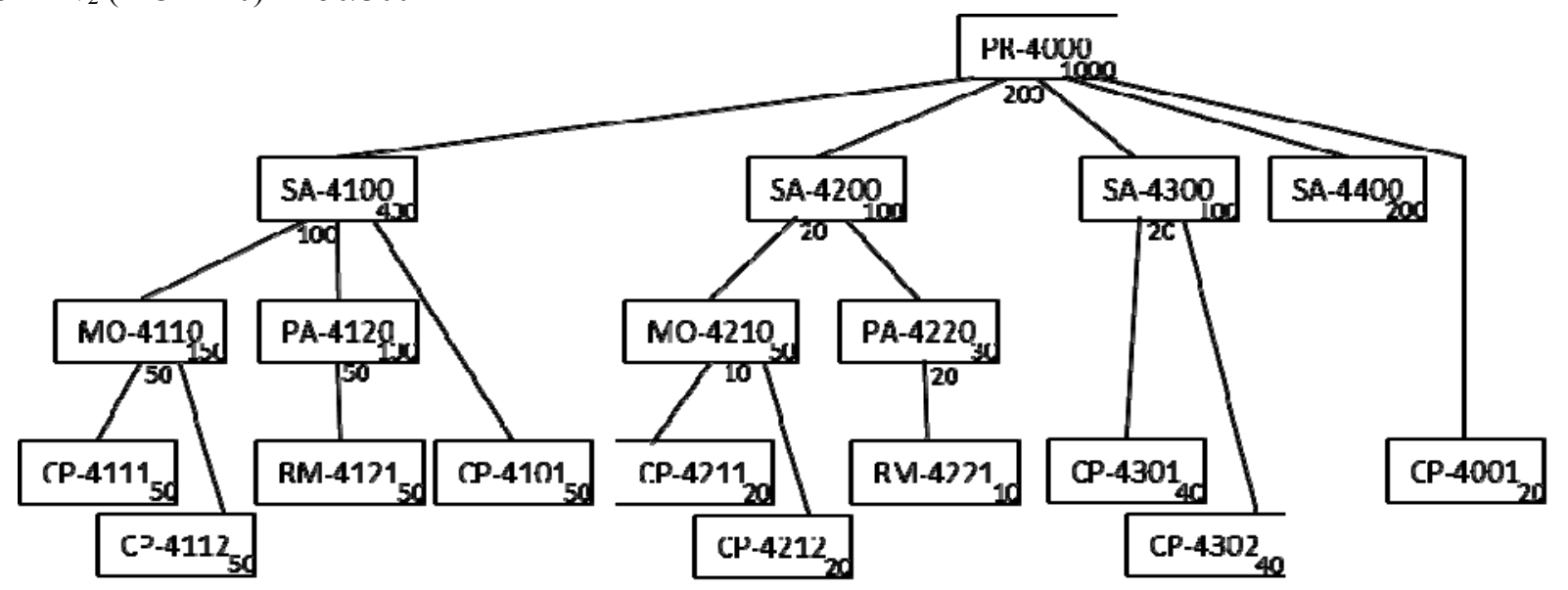

Figure 2 - Configuration Generic model with cost data 
could encompass this integration aspect.

The second issue that has not been considered in the previous sections is the "nature of change" on the GItems. By nature of change we mean whether the customer requirement is an interpolation or an extrapolation of existing items. For example, if we consider a crane product with a standard offer that can have three different heights: 6,10 and 14 meters. An out of range requirement of 12 meters would be an interpolation while that of 16 meters would be an extrapolation. In most of the cases, the ETO-Level of an extrapolation is higher than that of an interpolation.

This second issue is much more difficult to handle because it has sense to consider an ETO case that need the re-design of all G-Items of the G-BOM with an interpolation. In that case the ETO-L will be equal to 1000 , so what could be the ETO-L of an extrapolation? In fact, the nature of change is more a characteristic of a kind of risk about the ability of the supplier to design a feasible product that covers the out of range requirements. As a very first idea, we suggest to add a second metric to each G-item that would represent the risk relevant to the nature of change plus some aggregation mechanisms.

\section{CONCLUSION AND FURTHER RESEARCH}

In Engineering-To-Order configuration situations where customers' demands are out of the standard of the supplier companies, the goal of this article was to provide metrics that enable suppliers to answer the following questions: Do we accept the out of standard demands? How far are they from our standard? What are the risks?

After a recall of the configuration basics, we have assumed a four-level generic configuration model in order to define relevant metrics. Three approaches have been presented. The two first ones were essentially related to physical aspect and operate only with the analysis of the G-BOM. They are easy to implement and do not require any specific data. The only requirement is to identify the G-Items of the G-BOM that should be redesigned. The third one considers the relative importance of each GItem. As a first idea, we have considered the cost of the G-Items as their relative importance. We think that this third approach allows to build a metric which can provide with a moderate effort a good idea of the ETO-Level of any non-standard solution. We are currently working on two important issues (integration and nature of change) in order to propose more realistic metrics.

Once achieved, these metrics should greatly assist suppliers working in B2B in CTO situations and facing some out of standard demands. More precisely they will assist them to decide whether to respond or not to such a demand. Furthermore, if the supplier company stores data relevant to all non-standard offers with the required engineering workload, it should be possible to improve the confidence in the metric with some data analytics.

\section{ACKNOWLEDGMENT}

The authors would like to thank the French National Research Agency and the partners of the OPERA project.

\section{REFERENCES}

[1] A. Felfernig, L. Hotz, C. Baglay, and J. Tiihonen, Knowledge-based configuration From Research to Business Cases. 2014.

[2] Z. Siddique and J. a. Ninan, "Modeling of modularity and scaling for integration of customer in design of engineer-toorder products," Integr. Comput. Aided. Eng., vol. 13, no. 2, pp. 133-148, 2006.

[3] S. Mittal and F. Frayman, "Towards a generic model of configuration tasks," in Proceedings of the Eleventh International Joint Conference on Artificial Intelligence, 1989, vol. 2, pp. 1395-1401.

[4] T. Soininen, J. Tiihonen, T. Männistö, and R. Sulonen, "Towards a general ontology of configuration," Ai Edam, vol. 12 , no. 04, pp. 357-372, 1998.

[5] É. Vareilles, M. Aldanondo, and P. Gaborit, "Evaluation and design : a knowledge-based approach" Int. J. Comput. Integr. Manuf., vol. 3052, no. August, 2017.

[6] D. Yang and M. Dong, "Applying constraint satisfaction approach to solve product configuration problems with cardinality-based configuration rules," J. Intell. Manuf., pp. 99-111, 2013

[7] A. Sylla, D. Guillon, E. Vareilles, M. Aldanondo, T. Coudert, and L. Geneste, "Configuration knowledge modeling: How to extend con fi guration from assemble / make to order towards engineer to order for the bidding process," Comput. Ind., vol. 99, pp. 29-41, 2018.

[8] M. Aldanondo and E. Vareilles, "Configuration for mass customization: How to extend product configuration towards requirements and process configuration," J. Intell. Manuf., vol. 19, no. 5, pp. 521-535, 2008.

[9] E. Vareilles, A. F. B. Santa, M. Falcon, M. Aldanondo, and P. Gaborit, "Configuration of high performance apartment buildings renovation: A constraint based approach," IEEE Int. Conf. Ind. Eng. Eng. Manag., pp. 684-688, 2014.

[10] S. Shafiee, K. Kristjansdottir, and L. Hvam, "Automatic Identification of Similarities Across Products to Improve the Configuration Process in ETO Companies," Int. J. Ind. Eng. Manag., vol. 8, no. 3, pp. 167-176, 2017.

[11] H. Thevenot and T. Simpson, "Commonality indices for product family design: A detailed comparison," J. Eng. Des., vol. 17, no. 2, pp. 99-119, 2006.

[12] B. J. Sauser, J. E. R. Marquez, D. Henry, and D. DiMarzio, "A system maturity index for the systems engineering life cycle,” Int. J. Ind. Syst. Eng., vol. 3, no. 6, p. 673, 2008. 